\title{
Do Communication Patterns Affect the Association between Cognitive Impairment and Hearing Loss among Older Adults in Vietnam?
}

\author{
Tran Dai Tri Han ${ }^{1,2}\left(\mathbb{D}\right.$, Keiko Nakamura ${ }^{1, *(\mathbb{D}}$, Kaoruko Seino ${ }^{1}$, Vo Nu Hong Duc ${ }^{2}$ (D) and Thang Van Vo ${ }^{2,3}(\mathbb{D}$ \\ 1 Department of Global Health Entrepreneurship, Division of Public Health, Graduate School of Medical and \\ Dental Sciences, Tokyo Medical and Dental University, Tokyo 113-8519, Japan; \\ trandaitrihan@hueuni.edu.vn (T.D.T.H.); seino.ith@tmd.ac.jp (K.S.) \\ 2 Faculty of Public Health, Hue University of Medicine and Pharmacy, Hue University, Hue 530000, Vietnam; \\ vnhduc@hueuni.edu.vn (V.N.H.D.); vovanthang147@hueuni.edu.vn (T.V.V.) \\ 3 The Institute for Community Health Research, Hue University of Medicine and Pharmacy, Hue University, \\ Hue 530000, Vietnam \\ * Correspondence: nakamura.ith@tmd.ac.jp
}

Citation: Han, T.D.T.; Nakamura, K.; Seino, K.; Duc, V.N.H.; Vo, T.V. Do Communication Patterns Affect the Association between Cognitive Impairment and Hearing Loss among Older Adults in Vietnam?. Int. J. Environ. Res. Public Health 2021, 18, 1603. https://doi.org/10.3390/ ijerph18041603

Academic Editor: Telmo Pereira

Received: 28 December 2020

Accepted: 4 February 2021

Published: 8 February 2021

Publisher's Note: MDPI stays neutral with regard to jurisdictional claims in published maps and institutional affiliations.

Copyright: (c) 2021 by the authors. Licensee MDPI, Basel, Switzerland. This article is an open access article distributed under the terms and conditions of the Creative Commons Attribution (CC BY) license (https:/ / creativecommons.org/licenses/by/ $4.0 /)$.

\begin{abstract}
This study examined the prevalence of cognitive impairment among older adults in central Vietnam and the roles of communication (with or without communication devices) in the association between cognitive impairment and hearing loss. This cross-sectional study was performed on 725 randomly selected community-dwelling older adults aged $\geq 60$ years from Thua Thien Hue province, Vietnam. Participants attended a face-to-face survey. Sociodemographic characteristics, social interaction with or without communication devices, health status and cognitive function using the Mini-Mental State Examination were reported. Ordinal logistic regression analysis was performed to quantify the association between hearing loss and cognitive function by frequency of communication with and without devices. Mild and severe cognitive impairment had prevalence rates of $23.6 \%$ and $19.3 \%$, respectively. Cognitive impairment was more prevalent among older adults with hearing-loss, vision loss and difficulties with instrumental activities of daily living (IADL). The association between hearing loss and cognitive impairment was not significant when older adults had frequent communication with others using devices. This study presented the relatively high prevalence of cognitive impairment in community-dwelling older adults in Vietnam. Frequent communication using devices attenuated the association between hearing loss and cognitive impairment.
\end{abstract}

Keywords: communication tools; cognitive impairment; older adults; hearing loss; social interaction

\section{Introduction}

Promotion of cognitive health has been a public health priority in rapidly aging societies considering its impact not only on older adults' quality of life, but also on their families, health care system and economy [1]. Vietnam is one of the countries with fastest rate of aging in the world [2]. The number of people aged $\geq 65$ years in Vietnam was 7.4 million in 2019 , accounting for $7.7 \%$ of population [3], which is expected to increase to $18.1 \%$ by 2049 [4].

Hearing loss, the most prevalent sensory deficit affecting about one in three adults aged over 65 years [5], has not been a priority in cognitive impairment risk management for long [6]. However, cohort studies have recently showed that even mild levels of hearing loss increase the longer-term risk of cognitive decline $[7,8]$. However, reported results on the association between cognitive impairment and hearing loss are conflicting $[9,10]$ and the underlying mechanism to clearly explain these two associations is not yet established.

Hearing loss leads to difficulties in communication and creates barriers in interaction [11,12]. Social isolation, defined as having a small social network or a lack of close 
relationships or sources of social support [13], is one of the factors that relate both with cognitive impairment and hearing loss $[14,15]$. The development of information technology has diverted ways of communication from simple telephone calling to video calls or text messaging application, which allows declined visual and hearing function to be mutually complemented [16]. Easy-to-use options, including modulating pitch and amplitude on communication devices, enable older adults to compensate peripheral functional deficit of age related hearing loss [17]. In addition, communication device usage makes up for face-to-face communication limitation due to changes in family structure and lifestyles [18]. Considering the interrelationships of cognitive impairment, hearing loss, and interpersonal communication, the question emerges how usage of communication tools may affect the association between cognitive impairment and hearing loss.

In Vietnam, with the rapid development of information and communication technology, telephone and internet access have become more accessible and affordable. The percentage of internet users per 100 inhabitants in Vietnam exceeds $50 \%$, and the number of mobile cellular telephone subscribers is equal to the size of the Vietnamese population $[3,19]$. In $2019,91.7 \%$ of households owned phone or mobile phone or tablet (comparing to $45.7 \%$ in 2009) [3,20]. In addition, communication applying these technologies into usage of smartphones or social media has been penetrated even among older adults in communities where face to face communication with their children, relatives, and close friends is limited due to changes in family structure and lifestyles [21].

To date, there were few primary studies in Vietnam focused on the cognitive impairment of older adults. These studies mainly investigated epidemiological aspects of cognitive impairment. The previous Vietnam reports showed the prevalence of cognitive impairment ranged from $29 \%$ to $48 \%$ [22-24]. However, a lack of study included mild cognitive impairment (MCI), an early stage of dementia. Moreover, though much research has documented that hearing loss was associated with cognitive impairment in high-income countries, a minimal number of studies have been done in low- and middle-income countries, including Vietnam, and few have focused on the role of communication patterns. A better understanding of communication patterns' role in the association between hearing loss and cognitive impairment would provide valuable insights into potential approaches in preventing or delaying cognitive decline progress.

This study was performed to estimate the prevalence of cognitive impairment among older adults in central Vietnam and explore the effects of the use of communication devices on the association between hearing loss and cognitive impairment.

\section{Methods}

\subsection{Participants}

This cross-sectional investigation was conducted in Thua Thien Hue province in the central region of Vietnam between June and July 2018. A total of 725 community-dwelling adults aged $\geq 60$ years were selected by two-stage random sampling. In stage one of the sampling process, the population was divided into rural and urban areas. Two of 27 quarters in Hue city (urban areas) and three of 19 quarters of Phu Vang district (rural areas) were randomly selected. In stage two, the participants were proportionate randomly selected from lists of all potential participants in the five quarters. Participants who were unable to communicate were excluded.

Because the standardized cutoffs for cognitive impairment were developed primarily in Western countries and no normative studies have been conducted in Vietnam, use of the Western cutoffs is potentially problematic due to the inclusion of illiterate participants who would not be able to respond to questions requiring reading and writing ability. Therefore, participants who were illiterate were excluded $(n=81)$ to reduce misclassification errors in the present study. Evaluation of cognitive function by using the Mini Mental State Examination (MMSE) determined 81 potential participants ineligible to continue with the study. The remaining 644 literate participants were interviewed and their information analyzed. 


\subsection{Measurements}

The study participants attended a face-to-face interview with interviewers who were public health specialists with psychiatric knowledge. Questions designed in English were converted to Vietnamese by a forward and back translation process.

\subsubsection{Sociodemographic and Types of Interaction}

In addition to age and sex, level of education was categorized into elementary school or lower, secondary or high school, and university or higher. Living area was categorized as rural or urban. Living with spouse, and children were categorized as "yes" or "no." Financial strain was categorized as "no" when participants had enough money for their daily needs over the past month, or "yes" if they lacked money for daily needs.

Interaction using communication devices was defined as the use of tools of telephone, e-mail, or social media to communicate with family and others. Interaction not using communication devices was defined as face-to-face direct interactions with family and others at social gatherings, such as going out together or visiting each other's homes. Both interactions using and not using communication devices were classified as frequent ( $\geq 2$ times per week) or infrequent ( $<2$ times per week).

\subsubsection{Health Status and Lifestyles}

\section{Cognitive Function}

Cognitive function was evaluated using the MMSE [25], a paper-based test that is a commonly used standard instrument for detection of cognitive impairment $[26,27]$. The MMSE includes two parts, the first of which requires vocal responses and covers orientation to time and place, word repetition and recall, and attention, while the second part tests the ability to name objects, follow verbal and written commands, write sentences, and copy complex polygons. The MMSE total score ranges from 0 to 30, with higher scores indicating better cognitive function. Cognitive function evaluated by MMSE was divided into three categories: MMSE 0-23, MMSE 24-27, and MMSE 28-30 based on cutoff points of $23 / 24$ and $27 / 28$ reported to distinguish different older adults' cognition [27-29].

\section{Sensory Function}

A short questionnaire to assess self-perceived hearing and vision loss was administered at the interviews. In community surveys to cover a large population, this method is widely used, and several studies have shown that self-rated hearing impairment is correlated with audiometric measures in older adults [30,31]. Participants were asked to rate their hearing and vision ability as: no difficulty, difficult, or very difficult. Participants with hearing or vision ability classified as difficult or very difficult were categorized as having hearing or vision loss.

\section{Instrumental Activities of Daily Living (IADL)}

Eight items of the Lawton Instrumental Activities of Daily Living (IADL), i.e., using a telephone, shopping, food preparation, housekeeping, doing laundry, using transportation, taking medications, and financial behavior, were used to assess the ability of older adults participants to perform daily tasks [32]. Difficulty with IADL was defined as the inability to perform at least one of the above items independently [33].

\section{Presence of Chronic Disease}

The presence of chronic disease (angina, asthma, arthritis, cataracts, chronic obstructive pulmonary disease, diabetes, hypertension) was categorized as no, one, and more than one chronic disease based on self-reporting of clinical diagnosis history.

Smoking

Current smoking status was categorized as yes or no. 


\subsection{Statistical Analysis}

The frequencies of demographic and socioeconomic characteristics, interaction types, cognitive function, health status, living arrangement, and lifestyles of participants were calculated. The chi-square test was used to compare the levels of cognitive impairment according to the frequencies of interaction types, sociodemographic characteristics, health status, and lifestyles of participants. Ordinal logistic regression analysis was performed to quantify the associations between hearing loss and cognitive function outcomes according to the frequencies of interaction types, with adjustments for sociodemographic characteristics, health status, living arrangement, and lifestyle.

Ordinal logistic regression is the best fit when the dependent variable is ordinal and can be ordered in a natural way [34]. Odds ratio (OR) $>1$ indicates exposure associated with higher odds of decreased cognitive function.

Statistical analyses were performed using IBM SPSS Statistics version 25.0 (IBM, Armonk, NY, USA). In all analyses, $p<0.05$ was taken to indicate statistical significance.

\section{Results}

The characteristics of participants are shown in Table 1. The total number of study participants included in the statistical analysis was 644 literate adults aged 60 years and above, nearly half of whom were aged 60-69 years old, the majority were women (54.5\%), and more than a half had elementary school or lower educational level. Most subjects lived with a spouse or/and children. Self-rated hearing loss was documented in $21.9 \%$ of participants. There was no significant difference in the characteristics of participants between the original sample size $(n=725)$ and the sample of only literate participants $(n=644)$.

Table 1. Characteristics of study participants included in the analysis $(n=644)$.

\begin{tabular}{|c|c|c|}
\hline Variables & Number of Subjects & $(\%)$ \\
\hline \multicolumn{3}{|c|}{ Demographic and socio-economic status } \\
\hline \multicolumn{3}{|l|}{ Sex } \\
\hline Men & 293 & $(44.5)$ \\
\hline Women & 351 & $(54.5)$ \\
\hline \multicolumn{3}{|l|}{ Age group } \\
\hline $60-69$ & 313 & $(48.6)$ \\
\hline $70-79$ & 186 & $(28.9)$ \\
\hline$\geq 80$ & 145 & $(22.5)$ \\
\hline \multicolumn{3}{|l|}{ Education } \\
\hline Elementary school or lower & 337 & $(52.3)$ \\
\hline Secondary or high school & 251 & $(39.0)$ \\
\hline University or higher & 56 & $(8.7)$ \\
\hline \multicolumn{3}{|l|}{ Financial strain } \\
\hline No & 381 & $(59.2)$ \\
\hline Yes & 263 & $(40.8)$ \\
\hline \multicolumn{3}{|l|}{ Living area } \\
\hline Rural & 372 & $(57.8)$ \\
\hline Urban & 272 & $(42.2)$ \\
\hline \multicolumn{3}{|l|}{ Living with spouse } \\
\hline No & 168 & $(26.1)$ \\
\hline Yes & 476 & $(73.9)$ \\
\hline \multicolumn{3}{|l|}{ Living with children } \\
\hline No & 221 & $(34.3)$ \\
\hline Yes & 423 & $(65.7)$ \\
\hline
\end{tabular}


Table 1. Cont.

\begin{tabular}{|c|c|c|}
\hline Variables & Number of Subjects & $(\%)$ \\
\hline \multicolumn{3}{|c|}{ Health status and lifestyles } \\
\hline \multicolumn{3}{|l|}{ Vision loss } \\
\hline No & 343 & $(53.3)$ \\
\hline Yes & 301 & $(46.7)$ \\
\hline \multicolumn{3}{|l|}{ Hearing loss } \\
\hline No & 503 & $(78.1)$ \\
\hline Yes & 141 & $(21.9)$ \\
\hline \multicolumn{3}{|l|}{ IADL } \\
\hline Independence in IADL & 382 & $(59.3)$ \\
\hline Difficulty with IADL & 262 & $(40.7)$ \\
\hline \multicolumn{3}{|l|}{ Presence of chronic disease } \\
\hline None & 189 & $(29.3)$ \\
\hline One chronic disease & 246 & $(38.2)$ \\
\hline More than one chronic disease & 209 & $(32.5)$ \\
\hline \multicolumn{3}{|l|}{ Current smoking } \\
\hline Yes & 157 & $(24.4)$ \\
\hline No & 487 & $(75.6)$ \\
\hline \multicolumn{3}{|c|}{ Types of interaction } \\
\hline \multicolumn{3}{|c|}{ Interaction using communication devices ${ }^{1}$} \\
\hline Infrequent ( $<2$ times per week) & 306 & $(47.5)$ \\
\hline Frequent ( $\geq 2$ times per week) & 338 & $(52.5)$ \\
\hline \multicolumn{3}{|c|}{ Interaction not using communication devices ${ }^{2}$} \\
\hline Infrequent $(<2$ times per week) & 330 & $(51.2)$ \\
\hline Frequent ( $\geq 2$ times per week) & 314 & $(48.8)$ \\
\hline
\end{tabular}

Table 2 presents the levels of cognitive function according to the type of interactions, and the characteristics of the participants. Overall, $124(19.3 \%)$ participants were categorized as having severe cognitive impairment (MMSE 0-23), 152 (23.6\%) had mild cognitive impairment (MMSE 24-27), and 368 (57.1\%) had normal cognitive function (MMSE 2830). The prevalence rates of MMSE group scores were significantly different between participants with different frequencies of interaction types and hearing function $(p<0.05)$.

Table 3 shows the results of multivariable ordinal regression analysis of the association between hearing loss and cognitive function, stratified according to the frequencies of interaction using and not using communication devices. In the all combined model (model 1), cognitive impairment was associated with hearing loss, vision loss, being female, age over 80, lower education, current smoking, and difficulty with IADL. Model 2 showed association of hearing loss with cognitive impairment in participants with infrequent interactions using devices (OR: 2.23, 95\% CI: 1.26-3.93 $p=0.006$ ). However, association between hearing loss and cognitive impairment was attenuated in model 3 , and this relationship was not significant in participants with frequent interactions using communication devices (OR: 1.58, 95\% CI: 0.85-2.96, $p=0.148$ ). In models 4 and 5, self-rated hearing loss showed a significant association with cognitive impairment in participants with both infrequent and frequent interactions not using communication devices. Furthermore, vision loss, current smoker, and IADL difficulty showed significant associations with cognitive impairment regardless the frequencies of the interaction types. 
Table 2. Cognitive function levels according to types of interactions and the characteristics of participants ${ }^{1}$.

\begin{tabular}{|c|c|c|c|c|}
\hline \multirow[b]{2}{*}{ Variables } & \multicolumn{3}{|c|}{ No. (\%) by Cognitive Impairment } & \multirow[b]{2}{*}{$p$-Value } \\
\hline & $\begin{array}{l}\text { MMSE 0-23 } \\
\text { (Severe) } \\
(n=124)\end{array}$ & $\begin{array}{c}\text { MMSE 24-27 } \\
\text { (Mild) } \\
(n=152)\end{array}$ & $\begin{array}{l}\text { MMSE 28-30 } \\
\quad \begin{array}{l}\text { Normal) } \\
(n=368)\end{array}\end{array}$ & \\
\hline \multicolumn{5}{|c|}{ Types of interaction } \\
\hline $\begin{array}{c}\text { Interactions using communication dev } \\
\text { Infrequent ( }<2 \text { times per week) } \\
\text { Frequent ( } \geq 2 \text { times per week) }\end{array}$ & $\begin{array}{l}80(26.1) \\
44(13.0)\end{array}$ & $\begin{array}{l}71(23.2) \\
81(24.0)\end{array}$ & $\begin{array}{l}155(50.7) \\
213(63.0)\end{array}$ & $<0.001$ \\
\hline $\begin{array}{c}\text { Interactions not using communication d } \\
\text { Infrequent }(<2 \text { times per week) } \\
\text { Frequent }(\geq 2 \text { times per week })\end{array}$ & $\begin{array}{l}76(23.0) \\
48(15.3) \\
\end{array}$ & $\begin{array}{l}74(22.4) \\
78(24.8) \\
\end{array}$ & $\begin{array}{l}180(54.5) \\
188(59.9)\end{array}$ & 0.045 \\
\hline \multicolumn{5}{|c|}{ Demographic and socioeconomic status } \\
\hline $\begin{array}{l}\text { Sex } \\
\text { Women } \\
\text { Men }\end{array}$ & $\begin{array}{l}80(22.8) \\
44(15.0)\end{array}$ & $\begin{array}{l}92(26.2) \\
60(20.5) \\
\end{array}$ & $\begin{array}{l}179(51.0) \\
189(64.5)\end{array}$ & 0.002 \\
\hline $\begin{array}{l}\text { Age group } \\
\begin{array}{c}60 \\
70\end{array}=69 \\
\geq 80\end{array}$ & $\begin{array}{l}31(9.9) \\
31(16.7) \\
62(42.8)\end{array}$ & $\begin{array}{l}70(22.4) \\
45(24.2) \\
37(25.5)\end{array}$ & $\begin{array}{l}212(67.7) \\
110(59.1) \\
46(31.7)\end{array}$ & $<0.001$ \\
\hline $\begin{array}{c}\text { Education } \\
\text { Elementary school or lower } \\
\text { Secondary or high school } \\
\text { University or higher }\end{array}$ & $\begin{array}{l}99(29.4) \\
22(8.8) \\
3(5.4)\end{array}$ & $\begin{array}{l}96(28.5) \\
46(18.3) \\
10(17.9)\end{array}$ & $\begin{array}{c}142(42.1) \\
183(72.9) \\
43(76.8) \\
\end{array}$ & $<0.001$ \\
\hline $\begin{array}{c}\text { Financial strain } \\
\text { No } \\
\text { Yes }\end{array}$ & $\begin{array}{l}57(15.0) \\
67(25.5)\end{array}$ & $\begin{array}{l}88(23.1) \\
64(24.3)\end{array}$ & $\begin{array}{l}236(61.9) \\
132(50.2)\end{array}$ & 0.002 \\
\hline $\begin{array}{l}\text { Living area } \\
\text { Rural } \\
\text { Urban } \\
\end{array}$ & $\begin{array}{l}88(23.7) \\
36(13.2)\end{array}$ & $\begin{array}{l}90(24.2) \\
62(22.8)\end{array}$ & $\begin{array}{l}194(52.2) \\
174(64.0)\end{array}$ & 0.002 \\
\hline $\begin{array}{l}\text { Living with spouse } \\
\text { No } \\
\text { Yes }\end{array}$ & $\begin{array}{l}48(28.6) \\
76(16.0)\end{array}$ & $\begin{array}{c}48(28.6) \\
104(21.8)\end{array}$ & $\begin{array}{c}72(42.9) \\
296(62.2)\end{array}$ & $<0.001$ \\
\hline $\begin{array}{l}\text { Living with children } \\
\text { No } \\
\text { Yes }\end{array}$ & $\begin{array}{l}40(18.1) \\
84(19.9)\end{array}$ & $\begin{array}{l}55(24.9) \\
97(22.9)\end{array}$ & $\begin{array}{l}126(57.0) \\
242(57.2)\end{array}$ & 0.791 \\
\hline \multicolumn{5}{|c|}{ Health status and lifestyles } \\
\hline $\begin{array}{l}\text { Hearing loss } \\
\text { No } \\
\text { Yes }\end{array}$ & $\begin{array}{l}71(14.1) \\
53(37.6)\end{array}$ & $\begin{array}{c}113(22.5) \\
39(27.7)\end{array}$ & $\begin{array}{c}319(63.4) \\
49(34.8)\end{array}$ & $<0.001$ \\
\hline $\begin{array}{l}\text { Vision loss } \\
\text { No } \\
\text { Yes }\end{array}$ & $\begin{array}{l}37(10.8) \\
87(28.9)\end{array}$ & $\begin{array}{l}66(19.2) \\
86(28.6)\end{array}$ & $\begin{array}{l}240(70.0) \\
128(42.5)\end{array}$ & $<0.001$ \\
\hline $\begin{array}{c}\text { Current smoking } \\
\text { Yes } \\
\text { No }\end{array}$ & $\begin{array}{l}38(24.2) \\
86(17.7)\end{array}$ & $\begin{array}{c}43(27.4) \\
109(22.4)\end{array}$ & $\begin{array}{c}76(48.4) \\
292(60.0)\end{array}$ & 0.036 \\
\hline $\begin{array}{c}\text { Presence of chronic disease } \\
\text { No } \\
\text { One chronic disease } \\
\text { More than one }\end{array}$ & $\begin{array}{l}28(14.8) \\
43(17.5) \\
53(25.4)\end{array}$ & $\begin{array}{l}45(23.8) \\
66(26.8) \\
41(19.6)\end{array}$ & $\begin{array}{l}116(61.4) \\
137(55.7) \\
115(55.0)\end{array}$ & 0.045 \\
\hline $\begin{array}{c}\text { IADL } \\
\text { Independence in IADL } \\
\text { Difficulty with IADL }\end{array}$ & $\begin{array}{c}28(7.3) \\
96(36.6)\end{array}$ & $\begin{array}{l}74(19.4) \\
78(29.8)\end{array}$ & $\begin{array}{c}280(73.3) \\
88(33.6)\end{array}$ & $<0.001$ \\
\hline
\end{tabular}

${ }^{1}$ Percentages may not total 100 due to rounding; ${ }^{2}$ Interaction using communication devices was defined as the use of tools of telephone, e-mail, or social media to communicate with family and others; ${ }^{3}$ Interaction not using communication devices was defined as face-toface direct interactions with family and others at social gatherings, such as going out together or visiting each other's homes; MMSE, Mini-Mental State Exam; IADL, Instrumental Activities of Daily Living. 
Table 3. Multivariable ordinal logistic regression analyses of the associations between hearing loss and cognitive function.

\begin{tabular}{|c|c|c|c|c|c|c|c|c|c|c|}
\hline \multirow{3}{*}{ Variables } & \multirow{2}{*}{\multicolumn{2}{|c|}{$\begin{array}{l}\text { Model } 1 \\
\text { All } \\
(n=644)\end{array}$}} & \multicolumn{4}{|c|}{ Interaction Using Communication Devices ${ }^{1}$} & \multicolumn{4}{|c|}{ Interaction Not Using Communication Devices ${ }^{2}$} \\
\hline & & & \multicolumn{2}{|c|}{$\begin{array}{c}\text { Model 2 } \\
\text { Infrequent } \\
(<2 \text { Times per Week) } \\
(n=306)\end{array}$} & \multicolumn{2}{|c|}{$\begin{array}{c}\text { Model } 3 \\
\text { Frequent } \\
(\geq 2 \text { Times per Week) } \\
(n=338)\end{array}$} & \multicolumn{2}{|c|}{$\begin{array}{c}\text { Model } 4 \\
\text { Infrequent } \\
(<2 \text { Times per Week) } \\
(n=330)\end{array}$} & \multicolumn{2}{|c|}{$\begin{array}{c}\text { Model 5 } \\
\text { Frequent } \\
(\geq 2 \text { Times per Week }) \\
(n=314)\end{array}$} \\
\hline & $\begin{array}{c}\text { OR } \\
(95 \% \mathrm{CI})\end{array}$ & $p$-Value & $\begin{array}{c}\text { OR } \\
(95 \% \mathrm{CI})\end{array}$ & $p$-Value & $\begin{array}{c}\text { OR } \\
(95 \% \mathrm{CI})\end{array}$ & $p$-Value & $\begin{array}{c}\text { OR } \\
(95 \% \mathrm{CI})\end{array}$ & $p$-Value & $\begin{array}{c}\text { OR } \\
(95 \% \mathrm{CI})\end{array}$ & $p$-Value \\
\hline \multicolumn{11}{|c|}{ Health status and lifestyles } \\
\hline \multicolumn{11}{|l|}{ Self-rated hearing loss } \\
\hline No & 1 & & 1 & & 1 & & 1 & & 1 & \\
\hline Yes & $\begin{array}{c}1.96 \\
(1.30-2.95)\end{array}$ & 0.001 & $\begin{array}{c}2.23 \\
(1.26-3.93)\end{array}$ & 0.006 & $\begin{array}{c}1.58 \\
(0.85-2.96)\end{array}$ & 0.148 & $\begin{array}{c}1.81 \\
(1.02-3.21)\end{array}$ & 0.042 & $\begin{array}{c}1.95 \\
(1.05-3.60)\end{array}$ & 0.034 \\
\hline \multicolumn{11}{|l|}{ Self-rated vision loss } \\
\hline No & 1 & & 1 & & 1 & & 1 & & 1 & \\
\hline Yes & $\begin{array}{c}2.02 \\
(1.41-2.88)\end{array}$ & $<0.001$ & $\begin{array}{c}1.97 \\
(1.18-3.31)\end{array}$ & 0.010 & $\begin{array}{c}2.07 \\
(1.24-3.45)\end{array}$ & 0.006 & $\begin{array}{c}2.23 \\
(1.34-3.70)\end{array}$ & 0.002 & $\begin{array}{c}1.74 \\
(1.02-2.96)\end{array}$ & 0.041 \\
\hline \multicolumn{11}{|l|}{ IADL } \\
\hline Difficulty with IADL & $\begin{array}{c}2.76 \\
(1.88-4.05)\end{array}$ & $<0.001$ & $\begin{array}{c}3.67 \\
(2.08-6.48)\end{array}$ & $<0.001$ & $\begin{array}{c}2.02 \\
(1.16-3.52)\end{array}$ & 0.013 & $\begin{array}{c}3.32 \\
(1.88-5.88)\end{array}$ & $<0.001$ & $\begin{array}{c}2.48 \\
(1.44-4.26)\end{array}$ & 0.001 \\
\hline \multicolumn{11}{|l|}{ Presence of chronic disease } \\
\hline No & 1 & & 1 & & 1 & & 1 & & 1 & \\
\hline One chronic disease & $\begin{array}{c}1.27 \\
(0.83-1.95)\end{array}$ & 0.278 & $\begin{array}{c}1.28 \\
(0.69-2.40)\end{array}$ & 0.431 & $\begin{array}{c}1.40 \\
(0.75-2.61)\end{array}$ & 0.295 & $\begin{array}{c}1.44 \\
(0.77-2.69)\end{array}$ & 0.252 & $\begin{array}{c}1.01 \\
(0.55-1.84)\end{array}$ & 0.976 \\
\hline More than one & $\begin{array}{c}1.06 \\
(0.68-1.65)\end{array}$ & 0.812 & $\begin{array}{c}1.10 \\
(0.57-2.10)\end{array}$ & 0.778 & $\begin{array}{c}1.17 \\
(0.61-2.26)\end{array}$ & 0.630 & $\begin{array}{c}1.65 \\
(0.89-3.08)\end{array}$ & 0.113 & $\begin{array}{c}0.64 \\
(0.33-1.25)\end{array}$ & 0.191 \\
\hline \multicolumn{11}{|l|}{ Current smoking } \\
\hline No & 1 & & 1 & & 1 & & 1 & & 1 & \\
\hline Yes & $\begin{array}{c}2.60 \\
(1.70-3.97)\end{array}$ & $<0.001$ & $\begin{array}{c}2.44 \\
(1.32-4.51)\end{array}$ & 0.004 & $\begin{array}{c}2.58 \\
(1.42-4.69)\end{array}$ & 0.002 & $\begin{array}{c}2.63 \\
(1.39-4.99)\end{array}$ & 0.003 & $\begin{array}{c}2.68 \\
(1.50-4.79)\end{array}$ & 0.001 \\
\hline
\end{tabular}


Table 3. Cont.

\begin{tabular}{|c|c|c|c|c|c|c|c|c|c|c|}
\hline \multirow{3}{*}{ Variables } & \multirow{2}{*}{\multicolumn{2}{|c|}{$\begin{array}{l}\text { Model 1 } \\
\text { All } \\
(n=644)\end{array}$}} & \multicolumn{4}{|c|}{ Interaction Using Communication Devices ${ }^{1}$} & \multicolumn{4}{|c|}{ Interaction Not Using Communication Devices ${ }^{2}$} \\
\hline & & & \multicolumn{2}{|c|}{$\begin{array}{c}\text { Model } 2 \\
\text { Infrequent } \\
(<2 \text { Times per Week) } \\
(n=306)\end{array}$} & \multicolumn{2}{|c|}{$\begin{array}{c}\text { Model } 3 \\
\text { Frequent } \\
(\geq 2 \text { Times per Week }) \\
(n=338)\end{array}$} & \multicolumn{2}{|c|}{$\begin{array}{c}\text { Model } 4 \\
\text { Infrequent } \\
(<2 \text { Times per Week) } \\
(n=330)\end{array}$} & \multicolumn{2}{|c|}{$\begin{array}{c}\text { Model } 5 \\
\text { Frequent } \\
(\geq 2 \text { Times per Week }) \\
(n=314)\end{array}$} \\
\hline & $\begin{array}{c}\text { OR } \\
(95 \% \mathrm{CI})\end{array}$ & $p$-Value & $\begin{array}{c}\text { OR } \\
(95 \% \mathrm{CI})\end{array}$ & $p$-Value & $\begin{array}{c}\text { OR } \\
(95 \% \mathrm{CI})\end{array}$ & $p$-Value & $\begin{array}{c}\text { OR } \\
(95 \% \mathrm{CI})\end{array}$ & $p$-Value & $\begin{array}{c}\text { OR } \\
(95 \% \mathrm{CI})\end{array}$ & $p$-Value \\
\hline \multicolumn{11}{|c|}{ Demographic and socioeconomic status } \\
\hline \multicolumn{11}{|l|}{ Sex } \\
\hline Women & 1 & & 1 & & 1 & & 1 & & 1 & \\
\hline Men & $\begin{array}{c}0.54 \\
(0.36-0.82)\end{array}$ & 0.004 & $\begin{array}{c}0.52 \\
(0.29-0.94)\end{array}$ & 0.031 & $\begin{array}{c}0.60 \\
(0.33-1.08)\end{array}$ & 0.087 & $\begin{array}{c}0.54 \\
(0.30-0.98)\end{array}$ & 0.044 & $\begin{array}{c}0.50 \\
(0.28-0.91)\end{array}$ & 0.023 \\
\hline \multicolumn{11}{|l|}{ Age group } \\
\hline $60=69$ & 1 & & 1 & & 1 & & 1 & & 1 & \\
\hline $70=79$ & $\begin{array}{c}1.12 \\
(0.74-1.71)\end{array}$ & 0.589 & $\begin{array}{c}1.34 \\
(0.73-2.47)\end{array}$ & 0.344 & $\begin{array}{c}0.94 \\
(0.51-1.72)\end{array}$ & 0.842 & $\begin{array}{c}0.92 \\
(0.50-1.67)\end{array}$ & 0.775 & $\begin{array}{c}1.28 \\
(0.69-2.36)\end{array}$ & 0.434 \\
\hline$\geq 80$ & $\begin{array}{c}2.27 \\
(1.73-4.43)\end{array}$ & $<0.001$ & $\begin{array}{c}3.36 \\
(1.72-6.59)\end{array}$ & $<0.001$ & $\begin{array}{c}2.20 \\
(1.12-4.33)\end{array}$ & 0.022 & $\begin{array}{c}2.37 \\
(1.18-4.76)\end{array}$ & 0.015 & $\begin{array}{c}3.04 \\
(1.56-5.91)\end{array}$ & 0.001 \\
\hline \multicolumn{11}{|l|}{ Education } \\
\hline Elementary school or lower & 1 & & 1 & & 1 & & 1 & & 1 & \\
\hline Secondary or high school & $\begin{array}{c}0.40 \\
(0.27-0.60)\end{array}$ & $<0.001$ & $\begin{array}{c}0.48 \\
(0.27-0.85)\end{array}$ & 0.012 & $\begin{array}{c}0.30 \\
(0.17-0.54)\end{array}$ & $<0.001$ & $\begin{array}{c}0.33 \\
(0.19-0.58)\end{array}$ & $<0.001$ & $\begin{array}{c}0.46 \\
(0.26-0.82)\end{array}$ & 0.008 \\
\hline University or higher & $\begin{array}{c}0.43 \\
(0.20-0.90)\end{array}$ & 0.025 & $\begin{array}{c}0.46 \\
(0.13-1.64)\end{array}$ & 0.230 & $\begin{array}{c}0.34 \\
(0.13-0.89)\end{array}$ & 0.028 & $\begin{array}{c}0.47 \\
(0.16-1.35)\end{array}$ & 0.160 & $\begin{array}{c}0.42 \\
(0.14-1.24)\end{array}$ & 0.117 \\
\hline \multicolumn{11}{|l|}{ Financial strain } \\
\hline No & 1 & & 1 & & 1 & & 1 & & 1 & \\
\hline Yes & $\begin{array}{c}1.09 \\
(0.75-1.56)\end{array}$ & 0.661 & $\begin{array}{c}0.84 \\
(0.50-1.41)\end{array}$ & 0.518 & $\begin{array}{c}1.50 \\
(0.89-2.54)\end{array}$ & 0.131 & $\begin{array}{c}0.98 \\
(0.58-1.66)\end{array}$ & 0.945 & $\begin{array}{c}1.11 \\
(0.66-1.89)\end{array}$ & 0.690 \\
\hline \multicolumn{11}{|l|}{ Living area } \\
\hline Rural & 1 & & 1 & & 1 & & 1 & & 1 & \\
\hline Urban & $\begin{array}{c}0.75 \\
(0.51-1.09)\end{array}$ & 0.135 & $\begin{array}{c}0.75 \\
(0.43-1.30)\end{array}$ & 0.306 & $\begin{array}{c}0.83 \\
(0.48-1.54)\end{array}$ & 0.518 & $\begin{array}{c}0.88 \\
(0.52-1.50)\end{array}$ & 0.644 & $\begin{array}{c}0.63 \\
(0.35-1.11)\end{array}$ & 0.110 \\
\hline
\end{tabular}


Table 3. Cont.

\begin{tabular}{|c|c|c|c|c|c|c|c|c|c|c|}
\hline \multirow{3}{*}{ Variables } & \multirow{2}{*}{\multicolumn{2}{|c|}{$\begin{array}{l}\text { Model } 1 \\
\text { All } \\
(n=644)\end{array}$}} & \multicolumn{4}{|c|}{ Interaction Using Communication Devices ${ }^{1}$} & \multicolumn{4}{|c|}{ Interaction Not Using Communication Devices $^{2}$} \\
\hline & & & \multicolumn{2}{|c|}{$\begin{array}{c}\text { Model } 2 \\
\text { Infrequent } \\
(<2 \text { Times per Week) } \\
(n=306)\end{array}$} & \multicolumn{2}{|c|}{$\begin{array}{c}\text { Model } 3 \\
\text { Frequent } \\
(\geq 2 \text { Times per Week) } \\
(n=338)\end{array}$} & \multicolumn{2}{|c|}{$\begin{array}{c}\text { Model } 4 \\
\text { Infrequent } \\
(<2 \text { Times per Week) } \\
(n=330)\end{array}$} & \multicolumn{2}{|c|}{$\begin{array}{c}\text { Model } 5 \\
\text { Frequent } \\
(\geq 2 \text { Times per Week }) \\
(n=314)\end{array}$} \\
\hline & $\begin{array}{c}\text { OR } \\
(95 \% \text { CI })\end{array}$ & $p$-Value & $\begin{array}{c}\text { OR } \\
(95 \% \mathrm{CI})\end{array}$ & $p$-Value & $\begin{array}{c}\text { OR } \\
(95 \% \mathrm{CI})\end{array}$ & $p$-Value & $\begin{array}{c}\text { OR } \\
(95 \% \mathrm{CI})\end{array}$ & $p$-Value & $\begin{array}{c}\text { OR } \\
(95 \% \mathrm{CI})\end{array}$ & $p$-Value \\
\hline \multicolumn{11}{|l|}{ Living with spouse } \\
\hline Yes & $\begin{array}{c}0.90 \\
(0.60-1.35)\end{array}$ & 0.615 & $\begin{array}{c}0.89 \\
(0.49-1.59)\end{array}$ & 0.689 & $\begin{array}{c}0.97 \\
(0.54-1.74)\end{array}$ & 0.909 & $\begin{array}{c}0.52 \\
(0.28-0.94)\end{array}$ & 0.031 & $\begin{array}{c}1.46 \\
(0.82-2.60)\end{array}$ & 0.195 \\
\hline \multicolumn{11}{|l|}{ Living with children } \\
\hline No & 1 & & 1 & & 1 & & 1 & & 1 & \\
\hline Yes & $\begin{array}{c}1.06 \\
(0.73-1.52)\end{array}$ & 0.771 & $\begin{array}{c}0.93 \\
(0.55-1.56)\end{array}$ & 0.772 & $\begin{array}{c}1.26 \\
(0.75-2.13)\end{array}$ & 0.385 & $\begin{array}{c}0.99 \\
(0.59-1.66)\end{array}$ & 0.961 & $\begin{array}{c}1.06 \\
(0.63-1.81)\end{array}$ & 0.822 \\
\hline
\end{tabular}

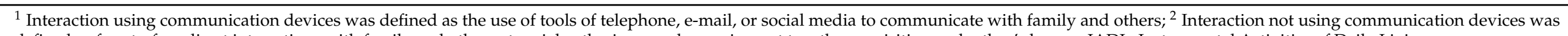
defined as face-to-face direct interactions with family and others at social gatherings, such as going out together or visiting each other's homes; IADL, Instrumental Activities of Daily Living. 


\section{Discussion}

Severe (MMSE 0-23) and mild (MMSE 24-27) cognitive impairment (MCI) had prevalence rates of $19.3 \%$ and $23.6 \%$, respectively, in our study population. The results presented here also suggested that the association hearing loss and cognitive function varied according to the frequencies and ways of communication. In particular, hearing loss was associated with decreased cognitive function among participants with infrequent interactions using devices and among those who had face-to-face interaction without devices regardless of the frequency. In contrast, frequent interactions using communication devices attenuated the association between hearing loss and cognitive impairment.

\subsection{Prevalence of Mild and Severe Cognitive Impairment}

In the present study, the combined prevalence of mild and severe cognitive impairment was $42.9 \%$, highlighting the public health impacts of these conditions and the need for national strategies to prevent cognitive impairment in older adults [35].

Based on 10 studies in eight countries the Cohort Studies of Memory in an International Consortium (COSMIC) showed that the prevalence of MCI ranged from $2.1 \%$ to $20.7 \%$ [36]. The COSMIC used the same criterion for MCI as in the present study (MMSE 24-27), but participants were people aged $\geq 65$ years and participants with dementia were excluded.

With regard to the prevalence of severe cognitive impairment, MMSE score 0-23 was used as the criterion to detect dementia and had sensitivity of 0.89 (95\% CI, 0.85 to 0.92$)$ and specificity of 0.89 ( $95 \%$ CI, 0.85 to 0.93 ) [26]. Using the same criterion of MMSE 0-23, but with some differences in methodologies and study populations, other studies in Vietnam reported severe cognitive impairment prevalence rates of $29-48 \%$ [22,24].

Furthermore, in our study, the combined prevalence of mild and severe cognitive impairment at $42.9 \%$ highlighted the public health impact of these conditions and the urgency for conducting national strategies to prevent or delay cognitive impairment in older adults [35].

\subsection{Diverse Association of Hearing Loss and Cognitive Function According to Interaction's Types}

The results of the present study showed cognitive impairment was more prevalent among older adults with hearing loss. Hearing loss has been recently recognized as a risk factor for dementia $[6,37,38]$. Hearing loss may be causally associated with cognitive impairment via increased cognitive load, changes in brain structure and function, and increased social isolation [14].

Our results showed that frequent interactions with family and others at social gatherings using communication devices attenuated the association between hearing loss and cognitive impairment. This may be explained by the attenuating effects of frequent interactions with communication supportive devices on social isolation in older adults with hearing loss, while the infrequent interactions or both frequent and infrequent interactions without using communication devices showed no such effect.

Direct interactions with family and others at social gatherings, such as going out together or visiting each other's homes not applying any communication device, are traditional ways to provide or receive social support. However, the direct interactions could bring challenges for older adults sometimes. Frequent interactions and dense social networks sometimes result in intrusive support, overwhelming advice and interference, and may exacerbate stress $[39,40]$. In particular, older adults may perceive well-intentioned support efforts from family or friends as control. Although such well-intentioned support can have beneficial effects on health outcomes, it can potentially lead to the development of interpersonal conflict and stress $[39,41]$. These aspects of the direct interactions without communication technology support may become more severe in older adults with hearing loss with partly limited communication ability.

In contrast, interactions involving the use of communication devices, such as telephone, e-mail, or social media, can somewhat offset the limitations of the direct interactions. Communication using devices are much easier to connect to a selected person regardless 
of time and geographical barriers, thus allowing older adults to gain access to the support that they need. In addition, with high rates of urbanization, older adults may have limited choice of living with a "significant family member or others." Indirect interactions with use of indirect means of communication allow older adults to choose the people with whom they connect. Although these selected individuals cannot provide as much instrumental support as the people who live with older adults, but they have the advantage of potentially providing better emotional support [42]. In addition, having the ability to use indirect means of communication, such as telephone, is beneficial for the self-efficacy of older adults [43].

In Vietnam, with the rapid development of information and communication technology, telephone and internet access have become more accessible and affordable. The percentage of internet users per 100 inhabitants in Vietnam exceeds 50\%, and the number of mobile cellular telephone subscribers is equal to the size of the Vietnamese population $[3,19]$. In the present study, $83 \%$ of older adults had the ability to use the telephone at different levels. These observations highlight the significant advantages of public health intervention programs based on interaction using communication devices. Noteworthy, communication means should be particularly designed for older adults who may have cognitive and functional limitations. Keeping in mind that even though some older adults do not have their own communication devices or face difficulties in learning how to use them, their housemates can operate communication devices for them.

The major strengths of this study included the use of population-based representative data, face-to-face interview, and standard data collection tools. Ordinal logistic regression allowed us to examine three levels of cognitive function as a dependent variable, including MCI, which is an early stage of cognitive decline that may have potential benefits for early preventive interventions. The results of this study revealed the critical role of interaction using communication devices, including telephone, mail, and online social networks, in modifying the association between hearing impairment and cognitive function.

This study had several limitations. First, due to its cross-sectional design, the results of associations of variables could not determine causal relationships. Further longitudinal studies are required to make causal inferences. Second, our results may have been subject to recall bias. If necessary, demographic information was double-checked with family members of the older adults included in the study. Third, the study relied on a self-reported measure of hearing loss. Our results were interpreted on the basis of understanding that self-rated hearing impairment is correlated with audiometric measures in older adults [31]. However, this correlation is still a matter of debate for cases of mild hearing impairment. Fourth, validity of MMSE in Vietnamese version to evaluate cognition of the Vietnamese population would be carefully examined in a future study. In addition, as MMSE includes two questions that evaluate reading and writing ability, illiterate participants $(n=81)$ were excluded from the analytical statistics to reduce misclassification of cognitive impairment by MMSE. This exclusion was helpful in analyzing the frequency of use of interaction using communication devices that require reading and writing ability, such as e-mail and social media. Therefore, our results should be interpreted in the context of the exclusion of illiterate participants.

Further longitudinal studies are required to elucidate the benefit of communication using devices on the association between hearing loss and cognitive impairment. Both quantitative and qualitative study designs are recommended. For example, a quantitative study could measure the impact of the specific means of communication on cognitive function in the specific context. A qualitative study could measure the advantages and disadvantages of using different means of communication from either perspective of older adults or their caregivers.

\section{Conclusions}

The association between cognitive impairment and hearing loss of older adults varied according to frequencies and ways of communication. Results of a study conducted in 
one of the world's fastest aging societies, Vietnam, showed that frequent communication using devices attenuated the association between hearing loss and cognitive impairment. These results suggested that fitted communication methods for older adults whose sensory functions declined with aging is vital to maintain their cognitive function.

Author Contributions: Conceptualization, T.D.T.H. and K.N.; methodology, T.D.T.H. and K.N.; software, T.D.T.H. and K.N; validation, K.N.; formal analysis, T.D.T.H. and K.N.; investigation, V.N.H.D. and T.V.V.; resources, K.N. and T.V.V.; data curation, T.D.T.H.; writing-original draft preparation, T.D.T.H. and K.N.; writing - review and editing, T.D.T.H., K.N., K.S., V.N.H.D., and T.V.V.; visualization, T.D.T.H., K.N., and K.S.; supervision, K.N.; project administration, K.N. and T.V.V.; funding acquisition, K.N. and T.V.V. All authors have read and agreed to the published version of the manuscript.

Funding: This research was funded by Institute for Community Health Research (ICHR)-Hue University of Medicine and Pharmacy (Hue UMP), the World Health Organization Centre for Health Development (WHO Kobe Centre-WKC: K18017), Scientific Research Grant, Ministry of Health, Labor and Welfare, Japan: 20GB1003, and Grant in Aid for Scientific Study, Japan Society for Promotion of Science, K19H04511.

Institutional Review Board Statement: The study was conducted according to the guidelines of the Declaration of Helsinki, approved by the Institutional Ethics Committee of Hue University of Medicine and Pharmacy, Vietnam (Approval number: H2018/148).

Informed Consent Statement: Informed consent was obtained from all subjects involved in the study.

Data Availability Statement: The data presented in this study are available on request from the corresponding author. The data are not publicly available due to them containing information that could compromise the privacy/consent of research participants.

Acknowledgments: The authors deeply thank all local organizations, provincial Department of Health, participants in Thua Thien Hue province, and staff members from the Institute for Community Health Research (ICHR) and from Faculty of Public Health of Hue University of Medicine and Pharmacy (Hue UMP) for data collection.

Conflicts of Interest: The authors declare no conflict of interest.

\section{References}

1. Prince, M.; Wimo, A.; Guerchet, M.; Ali, G.-C.; Wu, Y.-T.; Prina, M. World Alzheimer Report 2015. The Global Impact of Dementia. An Analysis of Prevalence, Incidence, Cost and Trends; Alzheimer's Disease International: London, UK, 2015.

2. United Nations ESCAP. Ageing in Asia and the Pacific: Overview; United Nations: Bangkok, Thailand, 2017.

3. Central Population and Housing Census Steering Committee. The Vietnam Population and Housing Census 2019; Statistical Publishing House: Hanoi, Vietnam, 2019.

4. General Statistics Office. Vietnam Population Projection 2014-2049; General Statistics Office, Ed.; Vietnam News Agency Publishing House: Hanoi, Vietnam, 2016.

5. Risk Reduction of Cognitive Decline and Dementia: WHO Guidelines; World Health Organization: Geneva, Switzerland, 2019.

6. Livingston, G.; Sommerlad, A.; Orgeta, V.; Costafreda, S.G.; Huntley, J.; Ames, D.; Ballard, C.; Banerjee, S.; Burns, A.; CohenMansfield, J.; et al. Dementia prevention, intervention, and care. Lancet 2017, 390, 2673-2734. [CrossRef]

7. Deal, J.A.; Sharrett, A.R.; Albert, M.S.; Coresh, J.; Mosley, T.H.; Knopman, D.; Wruck, L.M.; Lin, F.R. Hearing impairment and cognitive decline: A pilot study conducted within the atherosclerosis risk in communities neurocognitive study. Am. J. Epidemiol. 2015, 181, 680-690. [CrossRef]

8. Curhan, S.G.; Willett, W.C.; Grodstein, F.; Curhan, G.C. Longitudinal study of hearing loss and subjective cognitive function decline in men. Alzheimers Dement. 2019, 15, 525-533. [CrossRef] [PubMed]

9. Hong, T.; Mitchell, P.; Burlutsky, G.; Liew, G.; Wang, J.J. Visual Impairment, Hearing Loss and Cognitive Function in an Older Population: Longitudinal Findings from the Blue Mountains Eye Study. PLoS ONE 2016, 11, e0147646. [CrossRef] [PubMed]

10. Lin, M.Y.; Gutierrez, P.R.; Stone, K.L.; Yaffe, K.; Ensrud, K.E.; Fink, H.A.; Sarkisian, C.A.; Coleman, A.L.; Mangione, C.M. Vision impairment and combined vision and hearing impairment predict cognitive and functional decline in older women. J. Am. Geriatr. Soc. 2004, 52, 1996-2002. [CrossRef]

11. Chen, Y.R.; Schulz, P.J. The Effect of Information Communication Technology Interventions on Reducing Social Isolation in the Elderly: A Systematic Review. J. Med. Internet Res. 2016, 18, e18. [CrossRef] 
12. Strawbridge, W.J.; Wallhagen, M.I.; Shema, S.J.; Kaplan, G.A. Negative consequences of hearing impairment in old age: A longitudinal analysis. Gerontologist 2000, 40, 320-326. [CrossRef]

13. Mick, P.; Kawachi, I.; Lin, F.R. The association between hearing loss and social isolation in older adults. Otolaryngol. Head Neck Surg. 2014, 150, 378-384. [CrossRef] [PubMed]

14. Lin, F.R.; Albert, M. Hearing loss and dementia-Who is listening? Aging Ment. Health 2014, 18, 671-673. [CrossRef] [PubMed]

15. Yang, R.; Wang, H.; Edelman, L.S.; Tracy, E.L.; Demiris, G.; Sward, K.A.; Donaldson, G.W. Loneliness as a mediator of the impact of social isolation on cognitive functioning of Chinese older adults. Age Ageing 2020, 49, 599-604. [CrossRef]

16. Crowley, D.J.; Mitchell, D. Communication Theory Today; Stanford University Press: Palo Alto, CA, USA, 1994.

17. Kemper, S.; Lacal, J.C. Addressing the Communication Needs of an Aging Society. In Technology for Adaptive Aging; Pew, R.W., Hemel, S.B.V., Eds.; The National Academies Press: Washington, DC, USA, 2004. [CrossRef]

18. Procentese, F.; Gatti, F.; Di Napoli, I. Families and Social Media Use: The Role of Parents' Perceptions about Social Media Impact on Family Systems in the Relationship between Family Collective Efficacy and Open Communication. Int. J. Environ. Res. Public Health 2019, 16, 5006. [CrossRef] [PubMed]

19. Ministry of Information and Communication. White Book of Viet Nam Information and Communication Teachnology 2017; Information and Communication Publishing House: Hanoi, Vietnam, 2017.

20. Central Population and Housing Census Steering Committee. The 2009 Vietnam Population and Housing Census: Major Findings; Statistical Publishing House: Hanoi, Vietnam, 2010.

21. General Statistics Office. Chapter 4: Household Structure. In Vietnam Population and Housing Census 2009-Age-Sex Structure and Marital Status of the Population in Vietnam; General Statistics Office: Hanoi, Vietnam, 2011.

22. Bich, N.N.; Dung, N.T.T.; Vu, T.; Quy, L.T.; Tuan, N.A.; Binh, N.T.T.; Hung, N.T.; Anh, L.V. Dementia and associated factors among the elderly in Vietnam: A cross-sectional study. Int. J. Ment. Health Syst. 2019, 13, 57. [CrossRef] [PubMed]

23. Leggett, A.; Zarit, S.H.; Hoang, C.N.; Nguyen, H.T. Correlates of cognitive impairment in older Vietnamese. Aging Ment. Health 2013, 17, 915-923. [CrossRef]

24. Khanh, D.V.D.; Thang, V.V.; Dung, H.; Thang, T.B.; Tuyen, H.D.; Hue, H.D.; Duong, L.D. Prevalence of dementia among the elderly and health care needs for people living with dementia in an urban community of central Vietnam. Vietnam J. Public Health 2015, 3, 16-23.

25. Folstein, M.F.; Folstein, S.E.; McHugh, P.R. "Mini-mental state". A practical method for grading the cognitive state of patients for the clinician. J. Psychiatr. Res. 1975, 12, 189-198. [CrossRef]

26. Patnode, C.D.; Perdue, L.A.; Rossom, R.C.; Rushkin, M.C.; Redmond, N.; Thomas, R.G.; Lin, J.S. Screening for Cognitive Impairment in Older Adults: Updated Evidence Report and Systematic Review for the US Preventive Services Task Force. JAMA 2020, 323, 764-785. [CrossRef]

27. Creavin, S.T.; Wisniewski, S.; Noel-Storr, A.H.; Trevelyan, C.M.; Hampton, T.; Rayment, D.; Thom, V.M.; Nash, K.J.; Elhamoui, H.; Milligan, R.; et al. Mini-Mental State Examination (MMSE) for the detection of dementia in clinically unevaluated people aged 65 and over in community and primary care populations. Cochrane Database Syst. Rev. 2016, CD011145. [CrossRef] [PubMed]

28. Neale, R.; Brayne, C.; Johnson, A.L. Cognition and survival: An exploration in a large multicentre study of the population aged 65 years and over. Int. J. Epidemiol. 2001, 30, 1383-1388. [CrossRef] [PubMed]

29. Gussekloo, J.; Westendorp, R.G.; Remarque, E.J.; Lagaay, A.M.; Heeren, T.J.; Knook, D.L. Impact of mild cognitive impairment on survival in very elderly people: Cohort study. BMJ 1997, 315, 1053-1054. [CrossRef]

30. Ferrite, S.; Santana, V.S.; Marshall, S.W. Validity of self-reported hearing loss in adults: Performance of three single questions. Rev. Saude Publica 2011, 45, 824-830. [CrossRef]

31. Deepthi, R.; Kasthuri, A. Validation of the use of self-reported hearing loss and the Hearing Handicap Inventory for elderly among rural Indian elderly population. Arch. Gerontol. Geriatr. 2012, 55, 762-767. [CrossRef]

32. Lawton, M.P.; Brody, E.M. Assessment of Older People: Self-Maintaining and Instrumental Activities of Daily Living. Gerontologist 1969, 9, 179-186. [CrossRef] [PubMed]

33. Vittengl, J.R.; White, C.N.; McGovern, R.J.; Morton, B.J. Comparative validity of seven scoring systems for the instrumental activities of daily living scale in rural elders. Aging Ment. Health 2006, 10, 40-47. [CrossRef] [PubMed]

34. Bender, R.; Grouven, U. Ordinal logistic regression in medical research. J. R. Coll. Physicians Lond. 1997, 31, 546-551.

35. Nguyen, T.K.T.; Pham, L.T.; Dang, V.H.; Nguyen, H.L.; Tran, V.T.; Bales, S.; Dang, V.H. The Joint Annual Health Review 2016: Toward Healthy Aging in Vietnam; Medical Publishing House: Hanoi, Vietnam, 2018.

36. Sachdev, P.S.; Lipnicki, D.M.; Kochan, N.A.; Crawford, J.D.; Thalamuthu, A.; Andrews, G.; Brayne, C.; Matthews, F.E.; Stephan, B.C.M.; Lipton, R.B.; et al. The Prevalence of Mild Cognitive Impairment in Diverse Geographical and Ethnocultural Regions: The COSMIC Collaboration. PLoS ONE 2015, 10, e0142388. [CrossRef] [PubMed]

37. Michalowsky, B.; Hoffmann, W.; Kostev, K. Association Between Hearing and Vision Impairment and Risk of Dementia: Results of a Case-Control Study Based on Secondary Data. Front. Aging Neurosci. 2019, 11, 363. [CrossRef]

38. Deal, J.A.; Betz, J.; Yaffe, K.; Harris, T.; Purchase-Helzner, E.; Satterfield, S.; Pratt, S.; Govil, N.; Simonsick, E.M.; Lin, F.R. Hearing Impairment and Incident Dementia and Cognitive Decline in Older Adults: The Health ABC Study. J. Gerontol. A Biol. Sci. Med. Sci. 2017, 72, 703-709. [CrossRef]

39. Taylor, S.E. Social support: A review. In The Oxford Handbook of Health Psychology; Friedman, H.S., Ed.; Oxford University Press: New York, NY, USA, 2011; pp. 189-214. 
40. Fisher, E.B.; La Greca, A.M.; Greco, P.; Arfken, C.; Schneiderman, N. Directive and nondirective social support in diabetes management. Int. J. Behav. Med. 1997, 4, 131-144. [CrossRef]

41. Franks, M.M.; Stephens, M.A.P.; Rook, K.S.; Franklin, B.A.; Keteyian, S.J.; Artinian, N.T. Spouses' provision of health-related support and control to patients participating in cardiac rehabilitation. J. Fam. Psychol. 2006, 20, 311-318. [CrossRef]

42. Jiang, D.; Fung, H.H. Chapter 6-Social and Emotional Theories of Aging. In Work Across the Lifespan; Baltes, B.B., Rudolph, C.W., Zacher, H., Eds.; Academic Press: Cambridge, MA, USA, 2019; pp. 135-153. [CrossRef]

43. Roh, H.W.; Lee, Y.; Lee, K.S.; Chang, K.J.; Kim, J.; Lee, S.J.; Back, J.H.; Chung, Y.K.; Lim, K.Y.; Noh, J.S.; et al. Frequency of contact with non-cohabitating adult children and risk of depression in elderly: A community-based three-year longitudinal study in Korea. Arch. Gerontol. Geriatr. 2015, 60, 183-189. [CrossRef] 